\title{
Influence of Early Season Moxidectin Treatments on Acquisition of Immunity to Ostertagia ostertagi in Calves
}

Moxidectin, a newly developed endectocide, has previously been demonstrated to have a high efficacy against gastrointestinal nematodes in cattle (Ranjan et al. 1992, Scholl et al. 1992). In a field trial (Yang, Satrija and Nansen, unpublished data) matched groups of 8 first-season Black Pied Friesian heifer calves, 6-8 months of age, were turned out in early May 1992 on a permanent pasture contaminated with trichostrongyles. The groups were grazed separately on comparable plots until they were housed in mid October 1992. Group $B$ was treated with moxidectin at turnout, and group $\mathrm{C}$ was treated at turnout and again 8 weeks after turnout. A third group served as non-treated controls (group A). The results showed that the 2 early season prophylactic treatments (group C) reduced the late season herbage trichostrongyle larval infectivity with 80 to $90 \%$, whereas the reduction accomplished by only 1 treatment at turnout (group B) was much less. Group C excreted significantly lower numbers of strongyle eggs than groups $\mathrm{A}$ and $\mathrm{B}$. The non-treated controls showed clinical signs of trichostrongylosis in late September, which coincided with a steep rise in serum pepsinogen levels reaching an average peak level of 3.5 IU tyrosine litre $^{-1}$. A few animals in group B showed softening of faeces particularly in the very late season. In contrast, animals receiving 2 moxi- dectin treatments remained in a clinically good condition throughout the season.

It was of interest to evaluate whether or not the high reduction in the level of parasite exposure, as a result of the 2 treatments, might affect the development of immunity to trichostrongyles. This was assessed by giving the animals an experimental challenge infection with Ostertagia ostertagi 5 months after housing, i.e. in March of the following year (1993). In March, the animals in all groups were treated with twice the recommended dose of fenbendazole (Panacur ${ }^{\circledR}$, Hoechst) to remove the residing worms from the previous grazing season. Two weeks later, all animals were challenged with $200 O$. Ostertagi infective larvae $\left(\mathrm{L}_{3}\right)$ per $\mathrm{kg}$ body weight. The average body weight at this time was $440 \mathrm{~kg}$. The course of infection was monitored by taking faecal samples on week 0 and again on weeks $3,4,5$, and 6 after the challenge infection. Subsequently, faecal trichostrongyle egg counts and faecal larval cultivations were made. Upon challenge blood samples were taken at 1 week intervals, and serum pepsinogen levels were measured according to Ross et al. (1967). Softening of faeces was observed in individual animals from groups B and C on week 3 p.i. Otherwise no clinical signs of parasitic gastroenteritis were observed. Following challenge infection, the first strongyle eggs were 


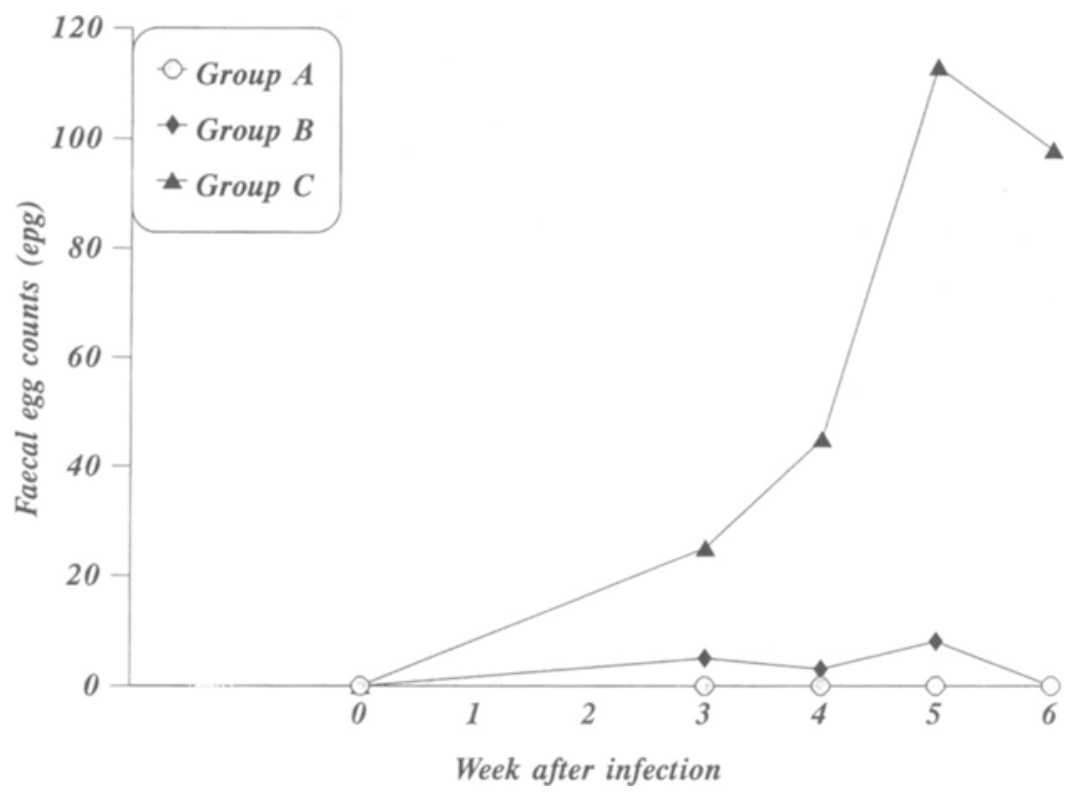

Figure 1. Mean trichostrongyle egg counts (epg) in the 3 experimental groups. Group A: previously nontreated animals; Group B: treated with moxidectin at turnout; Group C: treated with moxidectin at turnout and 8 weeks later.

detected in calves from groups $\mathrm{B}$ and $\mathrm{C}$ on week 3 p.i. (Fig. 1). Numbers of eggs excreted by group $\mathrm{C}$ calves rose steeply, reaching a peak of more than 100 epg on week 5 p.i. Only few eggs were found in the faeces of group B calves, and no trichostrongyle eggs were detected in the previously non-treated animals (group A). Faecal larval cultivation revealed that all eggs belonged to Ostertagia spp.

One week after infection, serum pepsinogen levels of all groups increased, whereafter they gradually declined towards the end of the experiment on week 6 p.i. (Fig. 2). No significant differences between groups were found. The non-treated controls tended to have higher serum pepsinogen concentrations at the start of the experiment despite the fact that these animals had previously been treated with fenbendazole. This may be due to the persistence of abomasal damage as a result of high worm burdens in the previous season (Chalmer, 1983). The early rise in the serum pepsinogen concentration (week 1 p.i.) may indicate an allergic reaction caused by the uptake of infective larvae (Armour et al. 1979).

The results of the present experiment suggest that early season prophylactic treatments with moxidectin given to calves during their first grazing season may negatively interfere with the development of acquired immunity to $O$. ostertagi in the subsequent year. This is evidenced by the absence of a patent $O$. ostertag $i$ infection upon the challenge of the previously non-treated controls, in contrast to the animals which had received moxidectin in the previous grazing season. The higher number of eggs excreted by animals receiving 2 moxidectin treatments (group C) compared with 1 


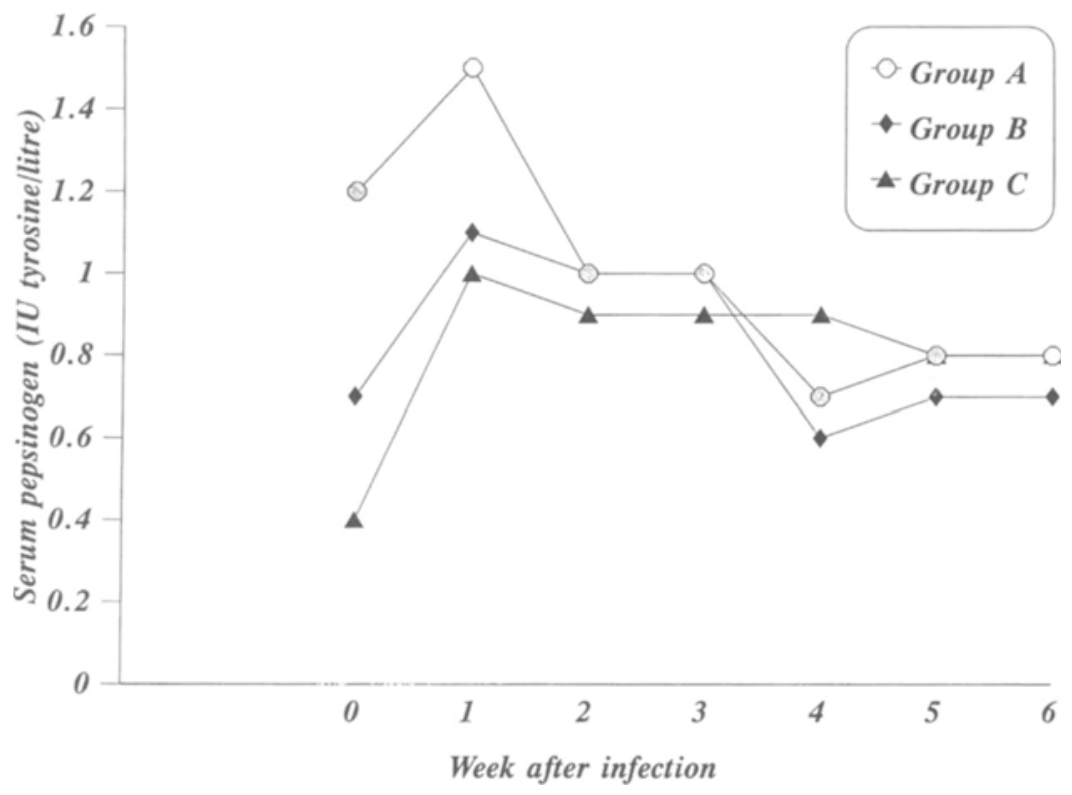

Figure 2. Mean serum pepsinogen levels (IU tyrosine litre ${ }^{-1}$ ) in the 3 experimental groups (see legend Fig. 1).

treatment (group B) indicates that the immunity in the former animals were more poorly developed. Jacobs et al. (1987) found similar results when first-season calves given an oxfendazole pulse release bolus were experimentally challenged in the housing period. Yet, the present results are not in accordance with Jacobs (1989) and Satrija (1993) who did not find any differences between previously ivermectin treated and non-treated secondseason heifers when these were naturally challenged during their second season grazing. This may be attributed to differences in the modes of challenge. Under natural conditions on pasture, second-season heifers are usually exposed to low levels of infection at the beginning of the season, a condition which is likely to boost the development of immunity before the animals encounter high pasture larval challenges in late summer (Jacobs et al. 1987). Such a build-up of immunity does not take place during the housing period, leaving the previously treated animals more susceptible to experimental challenge.

The lowered level of immunity in previously moxidectin treated heifers, demonstrated in this study, may only have potential practical implications in the very rare seasons where animals are confronted with heavy pasture loads of overwintered larvae already at the time of turnout (Nansen et al. 1989).

F. Satrija ${ }^{1,2}$, P. Nansen ${ }^{1}$, N. Midtgaard ${ }^{1}$ and X. Yang ${ }^{1}$ ${ }^{1}$ Danish Centre for Experimental Parasitology, The Royal Veterinary and Agricultural University, Frederiksberg, Denmark.

${ }^{2}$ Department of Parasitology and Pathology, Faculty of Veterinary Medicine, Bogor Agricultural University, Indonesia. 


\section{References}

Armour J, Bairden K, Duncan JL, Jennings FW, Parkins JJ: Observations on ostertagiasis in young cattle over two grazing seasons with special reference to plasma pepsinogen levels. Vet. Rec. 1979, 105, 500-503.

Chalmer K: Serum pepsinogen levels and Ostertagia ostertagi population in clinically normal adult beef cattle. N.Z. vet. J. 1983, 31, 189-191.

Jacobs DE, Pitt SR, Foster J, Fox MT: Interactions between chemoprophylaxis and immunity to bovine parasitic gastroenteritis and bronchitis: pilot studies using an oxfendazole pulse release blus. Res. vet. Sci. 1987, 43, 273-275.

Jacobs DE, Foster J, Gowling G, Pilkington JG, Fox $M T$, Ryan MT: Comparative study of early-season prophylaxis using ivermectin with lungworm vaccination in the control of parasitic bronchitis and gastroenteritis in cattle. Vet. Parasitol. 1989, 34, 45-56.

Nansen P, Grønvold J, Jørgensen RJ, Henriksen SAa, Foldager J, Sejrsen K: Outbreaks of early-season trichostrongylosis in calves in Denmark. Vet. Parasitol. 1989, 32, 199-211.

Ranjan S, Trudeau C, Prichard RK, Kutzleben $R$ von, Carrier $D$ : Efficacy of moxidectin against naturally acquired nematode infections in cattle. Vet. Parasitol. 1992, 41, 227-231.

Ross JG, Purcell DA, Dow C, Todd JR: Experimental infection of calves with Trichostrongylus axei: The course of development of infection and lesions in low level infections. Res. vet. Sci. 1967,8, 201-206.

Satrija $F$ : The effect of avermectin treatment on performance and immunity in calves during their first and second season grazing. Royal Veterinary and Agricultural University, Copenhagen. 1993. Ph.D. Thesis, pp. 159.

Scholl PJ, Guillot FS, Wang GT: Moxidectin: systemic activity against common cattle grups (Hypoderma lineatum) (Diptera: Oestridae) and trochostrongyle nematodes in cattle. Vet. Parasitol. 1992, 41, 203-209.

(Received February 20, 1995; accepted April 7, 1995).

Reprints may be obtained from: P. Nansen, Danish Centre for Experimental Parasitology, The Royal Veterinary and Agricultural University, Bülowsvej 13, DK-1870 Frederiksberg C, Denmark. 